\title{
Functor Categories and Two-Level Languages
}

\author{
E. Moggi
}

DISI - Univ. di Genova, via Dodecaneso 35,16146 Genova, Italy

phone: +39 10 353-6629, fax: +39 10 353-6699, e-mail: moggi@disi.unige.it

\begin{abstract}
We propose a denotational semantics for the two-level language of [GJ91, Gom92], and prove its correctness w.r.t. a standard denotational semantics. Other researchers (see [Gom91, GJ91, Gom92, JGS93, HM94]) have claimed correctness for lambda-mix (or extensions of it) based on denotational models, but the proofs of such claims rely on imprecise definitions and are basically flawed. At a technical level there are two important differences between our model and more naive models in Cpo: the domain for interpreting dynamic expressions is more abstract (we interpret code as $\lambda$-terms modulo $\alpha$-conversion), the semantics of newname is handled differently (we exploit functor categories). The key idea is to interpret a two-level language in a suitable functor category $\mathrm{Cpo}^{D^{a p}}$ rather than $\mathrm{Cpo}$. The semantics of newname follows the ideas pioneered by Oles and Reynolds for modeling the stack discipline of Algol-like languages. Indeed, we can think of the objects of $\mathcal{D}$ (i.e. the natural numbers) as the states of a name counter, which is incremented when entering the body of a $\lambda$-abstraction and decremented when coming out. Correctness is proved using Kripke logical relations (see [MM91, NN92]).
\end{abstract}

\section{Introduction}

Two-level languages are an important tool for analyzing programs. In the context of partial evaluation they are used to identify those parts of the program that can be reduced statically, and those that have to be evaluated dynamically. We take as representative of these two-level languages that described in [GJ91], which we call $P C F_{2}$, since it can be considered as the " $P C F$ of two-level languages". The main aims of this paper are: to point out the flaws in the semantics and correctness proof given in [Gom92], and to propose an alternative semantics for which one can prove correctness.

The interpretation of dynamic $\lambda$-abstraction given in [GJ91, Gom92] uses a newname construct "informally". Indeed, Gomard and Jones warn that "the generation of new variable names relies on a side-effect on a global state (a name counter). In principle this could have been avoided by adding an extra parameter to the semantic function, but for the sake of notational simplicity we use a less formal solution". Because of this informality, [GJ91, Gom92] are able to use a simplified semantic domain for dynamic expressions, but have to hand wave when it comes to the clause for dynamic $\lambda$-abstraction. This informality is maintained also in the correctness proof of [Gom92]. It is possible to fix the informal semantics using a name-counter (as suggested by Gomard and Jones), but then 
it is unclear how to fix the correctness proof. In fact, several experts were unable to propose a patch. Lack of precision in the definition of denotational semantics and consequent flaws in correctness proofs are not confined to [Gom92], indeed

- Chapter 4 of [Gom91] and Chapter 8 of [JGS93] contain the same definitions, results and proofs

- [GJ91] quotes the same definitions and results (but without proofs)

- while [HM94] adapts Gomard's technique to establish correctness for a polymorphic binding-time analysis (and introduces further flaws in the denotational semantics).

The specific model we propose is based on a functor category. In denotational semantics functor categories have been advocated by [Ole85] to model Algollike languages, and more generally they have been used to model locality and dynamic creation (see [OT92, PS93, FMS96]). For this kind of modeling they outperform the more traditional category Cpo of cpos (i.e. posets with lubs of $\omega$-chains and $\omega$-continuous maps). Therefore, they are a natural candidate for modeling the newname construct of [GJ91].

In the proposed functor category model the domain of residual programs is a bit more abstract than expected, namely $\alpha$-convertible programs are identified. This identification is necessary for defining the category $\mathcal{D}$ of dynamic expressions, but it seems also a desirable abstraction. Functor categories are definitely more complex than Cpo, but one can avoid most of the complexities by working in a metalanguage (with computational types). Indeed, it is only in few critical places, where it is important to know which category (and which monad) is used. The graduate textbook [Ten91] gives the necessary background on functor categories for denotational semantics to understand our functor category model. In Cpo models the renaming of bound dynamic variables (used in the interpretation of dynamic $\lambda$-abstraction) is modeled via a side-effect monad with a name-counter as state, on the contrary in the functor category model renaming is handled by the functor category itself (while non-termination at specialization-time is modeled by the lifting monad).

The paper is organized as follows: Section 1 recall the two-level language of [GJ91, Gom92] which we call $P C F_{2}$; Section 2 describes a general way for interpreting $P C F_{2}$ via translation into a metalanguage with computational types, and explains what's wrong with previously proposed semantics of $P C F_{2}$; Section 3 describes our functor category model for $P C F_{2}$ and proves correctness; Section 4 make a comparison of the semantics.

Acknowledgments. I wish to thank Olivier Danvy and Neil Jones for e-mail discussions, which were very valuable to clarify the intended semantics in [GJ91, Gom92], and to identify the critical problem in the correctness proof. Neil Jones has kindly provided useful bibliographic references and made available relevant internal reports. 


\section{The two-level language of Gomard and Jones}

In this section we recall the main definitions in [GJ91, Gom92], namely: the untyped object language $\lambda_{o}$ and its semantics, the two-level language $P C F_{2}$ and its semantics (including with the problematic clause for $\underline{\lambda}$ ). Both semantics are given via a translation into a metalanguage with computational type (see [Mog91, Mog97b]). In the case of $\lambda_{o}$ the monad corresponds to dynamic computations, while in the case of $P C F_{2}$ it corresponds to static computations.

\subsection{The untyped object language}

The object language $\lambda_{0}$ is an untyped $\lambda$-calculus with a set of ground constants $c$ (which includes the truth values)

$$
M::=x|\lambda x . M| M_{1} @ M_{2} \mid \text { fix } M \mid \text { if } M_{1} M_{2} M_{3} \mid c
$$

There is a canonical CBN interpretation of $\lambda_{0}$ in $D=(\text { const }+(D \rightarrow D))_{\perp}$, where const is the flat cpo of ground constants (ordered by equality). This interpretation can be described via a CBN translation ${ }^{n}$ into a suitable metalanguage with computational types, s.t. a $\lambda_{o}$-term $M$ is translated into a meta-term $M^{n}$ of type $T V$ with $V=$ const $+(T V \rightarrow T V)$, or more precisely $\bar{x}: T V \vdash_{M L} M^{n}: T V$ when $M$ is a $\lambda_{0}$-term with free variables included in the sequence $\bar{x}$ :

$-x^{n}=x$

$-c^{n}=[\operatorname{inl}(c)]$

$-(\lambda x \cdot M)^{n}=\left[\operatorname{inr}\left(\lambda x: T V \cdot M^{n}\right)\right]$

- $\left(M_{1} @ M_{2}\right)^{n}=$ let $u \Leftarrow M_{1}{ }^{n}$ in case $u$ of $\operatorname{inr}(f) \Rightarrow f\left(M_{2}{ }^{n}\right)$

$$
\Rightarrow \perp
$$

where $\perp: T V$ is the least element of $T V$

- (if $\left.M M_{1} M_{2}\right)^{n}=$ let $u \Leftarrow M^{n}$ in case $u$ of $\quad$ inl(true) $\Rightarrow M_{1}{ }^{n}$

$$
\begin{aligned}
\text { inl }(\text { false }) & \Rightarrow M_{2}{ }^{n} \\
& \Rightarrow \perp
\end{aligned}
$$

- $(\text { fix } M)^{n}=$ let $u \Leftarrow M^{n}$ in case $u$ of $\operatorname{inr}(f) \Rightarrow Y(f)$

$$
-\Rightarrow \perp
$$

where $Y:(T V \rightarrow T V) \rightarrow T V$ is the least fixed-point of $T V$

Note 1. $T$ can be any strong monad on Cpo s.t.: (i) each $T X$ has a bottom element $\perp$; (ii) let $x \Leftarrow \perp$ in $e=\perp$, i.e. $\perp$ is preserved by $f^{*}: T X \rightarrow T Y$ for any $f: X \rightarrow T Y$. With these properties one can interpret recursive definitions of programs and solve domain equations involving $T$.

The interpretation by Gomard amounts to take $T X=X_{\perp}$ and $D=T V$. 


\subsection{The two-level language $P C F_{2}$}

The two-level language $P C F_{2}$ can be described as a simply typed $\lambda$-calculus over the base types base and code with additional operations. The raw syntax of $P C F_{2}$ is given by

$$
\begin{aligned}
\text { - types } \tau::= & \text { base } \mid \text { code } \mid \tau_{1} \rightarrow \tau_{2} \\
\text { - terms } e::= & x|\lambda x: \tau . e| e_{1} @ e_{2}\left|f i x_{\tau} e\right| i f_{\tau} e_{1} e_{2} e_{3}|c| \\
& \text { lift } e|\underline{\lambda} x . e| e_{1} @ e_{2}|\underline{\text { fix }} e| \underline{\text { if } e_{1} e_{2} e_{3} \mid \underline{c}}
\end{aligned}
$$

The well-formed terms of $P C F_{2}$ are determined by assigning types to constants:

$-f_{i x_{\tau}}:(\tau \rightarrow \tau) \rightarrow \tau$

- $i f_{\tau}:$ base, $\tau, \tau \rightarrow \tau$

- $c:$ base

- lift : base $\rightarrow$ code

- $\underline{\lambda}:($ code $\rightarrow$ code $) \rightarrow$ code, following Church we have taken $\underline{\lambda}$ to be a higher order constant rather than a binder (all the binding is done by $\lambda$ ). The two presentations are equivalent: the term $\underline{\lambda}$ x.e of [GJ91] can be replaced by $\underline{\lambda}(\lambda x:$ code.e), while the constant $\underline{\lambda}$ can be defined as $\lambda f:$ code $\rightarrow$ code. $\underline{\lambda} x . f @ x$.

- @ : code, code $\rightarrow$ code

- fix : code $\rightarrow$ code

- if : code, code, code $\rightarrow$ code

- c: code

Remark. The language $P C F_{2}$ corresponds to the well-annotated expressions of Gomard and Jones. For two-level languages with dynamic type constructors (e.g. that in [HM94]) it is necessary to distinguish between static and dynamic types. In $P C F_{2}$ the only dynamic type is code, and there is no need to make this explicit.

\section{Models of $\mathrm{PCF}_{2}$ in Cpo}

The interpretation of $P C F_{2}$ is described by a translation ${ }_{-}^{s}$ into a suitable metalanguage with computational types, s.t. $x_{1}: \tau_{1}{ }^{s}, \ldots, x_{n}: \tau_{n}{ }^{s} \vdash_{M L} e^{s}: \tau^{s}$ when $x_{1}: \tau_{1}, \ldots, x_{n}: \tau_{n} \vdash_{P C F_{2}} e: \tau$. The translation highlights that static computations take place only at ground types (just like in $P C F$ and Algol).

- base $e^{s}=T$ (const), where const is the flat cpo of ground constants

- $\operatorname{code}^{s}=T(\exp )$, where $\exp$ is the flat cpo of open $\lambda_{0}$-terms with (free and bound) variables included in var $=\left\{x_{n} \mid n \in N\right\}$. When translating terms of $P C F_{2}$ we make use of the following expression-building operations:

- build_const : const $\rightarrow \exp$ is the inclusion of ground constants into terms

- build_var : var $\rightarrow \exp$ is the inclusion of variables into terms.

- build_@ : exp,exp $\rightarrow$ exp is the function $M_{1}, M_{2} \mapsto M_{1} @ M_{2}$ which builds an application. There are similar definitions for build_fix and build_if. 
- build $\lambda$ : var, exp $\rightarrow \exp$ is the function $x, M \mapsto \lambda x . M$ which builds a $\lambda$-abstraction.

$-\left(\tau_{1} \rightarrow \tau_{2}\right)^{s}=\tau_{1}^{s} \rightarrow \tau_{2}^{s}$

$-x^{s}=x$

$-c^{s}=[c]$

$-(\lambda x: \tau . e)^{s}=\lambda x: \tau^{s} \cdot e^{s}$

$-\left(e_{1} @ e_{2}\right)^{s}=e_{1}{ }^{s} @ e_{2}{ }^{s}$

- $\left(i f_{\tau} \text { e e } e_{1} e_{2}\right)^{s}=$ let $u \Leftarrow e^{s}$ in case $u$ of true $\Rightarrow e_{1}^{s}$

$$
\begin{aligned}
\text { false } & \Rightarrow e_{2}{ }^{3} \\
& \Rightarrow \perp
\end{aligned}
$$

where $\perp$ is the least element of $\tau^{s}$

- $\left(f i x_{\tau} e\right)^{s}=Y\left(e^{s}\right)$, where $Y$ is the least fixed-point of $\tau^{s}$

- $(\text { lift } e)^{s}=$ let $x \Leftarrow e^{s}$ in [build_const $\left.(x)\right]$

$-c^{s}=[$ build_const $(c)]$

- $(\underline{o p} \bar{e})^{s}=$ let $\bar{M} \Leftarrow \bar{e}^{s}$ in [build_op $\left.\bar{M}\right]$, where op $\in\{$ fix, @, if $\}$

* $(\underline{\underline{\lambda}} e)^{s}=$ let $x \Leftarrow$ newname in let $M \Leftarrow e^{s}([$ build_var $(x)])$ in [build_A $\left.(x, M)\right]$ where newname :T(var) generates a fresh variable of the object language.

The monad $T$ for static computations should satisfy the same additional properties stated in Note 1.

Remark. In the above interpretation/translation the meaning of newname (and $\underline{\lambda}$ ) is not fully defined, indeed one should fix first the interpretation of computational types $T X$.

The interpretation of [GJ91, Gom92] uses simplified semantic domains (which amount to use the lifting monad $T X=X_{\perp}$ ), but with these domains there is no way of interpreting newname (consistently with the informal description). Therefore, most of the stated results and proofs are inherently faulty.

Gomard and Jones are aware of the problem and say that "the generation of new variables names relies on a side effect on a global state (a name-counter)... but for the sake of notational simplicity we have used a less formal solution". Their proposed solution amounts to use a side-effect monad $T X=(X \times N)_{\perp}^{N}$, and to interpret newname $: T(v a r)$ as newname $=\lambda n: N \cdot u p\left(\left\langle x_{n}, n+1\right\rangle\right)$, where $u p_{X}: X \rightarrow X_{\perp}$ is the inclusion of $X$ into its lifting.

A simpler solution, suggested by Olivier Danvy, uses a state-reader monad $T X=$ $X_{\perp}^{N}$. In this case one can interpret the operation newname' $X_{X}:(T X)^{v a r} \rightarrow T X$ as newname ${ }_{X}^{\prime}(f)=\lambda n: N . f x_{n}(n+1)$, and use it for translating $\underline{\lambda}$

$$
\text { - }(\underline{\lambda} e)^{s}=\text { newname } e_{e x p}^{\prime}\left(\lambda x: \text { var.let } M \Leftarrow e^{s}([\text { build_var }(x)]) \text { in }[\text { build_ } \lambda(x, M)]\right) .
$$

The only place where a name-counter is really needed is for generating code, so we could use the simpler translation base $=$ const $_{\perp}$ and code $e^{s}=T(e x p)$. This is similar to what happens in Algol, where expressions cannot have side-effects, while commands can. 


\subsection{Correctness: attempts and failures}

Informally speaking, correctness for $P C F_{2}$ should say that for any $\emptyset \vdash_{P C F_{2}} e$ : code if the static evaluation of $e$ terminates and produces a $\lambda_{o}$-term $M: \exp$, then $\lambda_{0}$-terms $M$ and $e^{\phi}$ are equivalent, where ${ }_{-}^{\phi}$ is the translation from $P C F_{2}$ to $\lambda_{0}$ erasing types and annotations. In fact, this is an over-simplified statement, since one want to consider $P C F_{2}$-terms $\bar{x}:$ code $\vdash_{P_{C F}} e$ : code with free dynamic variables.

In a denotational setting one could prove correctness by defining a logical relation (see [MW85, Mit96]) between two interpretations of $P C F_{2}$

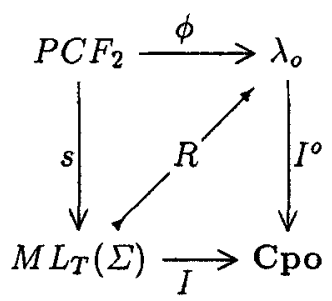

The parameterized logical relation $R_{\rho}^{r} \subseteq \llbracket \tau^{s} \rrbracket \times D$, where $\rho: v a r \rightarrow D$, proposed by [Gom92] is defined as follows

$-\perp R_{\rho}^{\text {base }} d$ and $u p(b) R_{\rho}^{\text {base }} d \stackrel{\Delta}{\Longleftrightarrow} d=u p\left(\operatorname{in}_{1} b\right)$

$-\perp R_{\rho}^{\text {code }} d$ and $u p(M) R_{\rho}^{\text {code }} d \stackrel{\Delta}{\Leftrightarrow} d=\llbracket M \rrbracket_{\rho}^{\circ}$

- $f R_{\rho}^{\tau_{1} \rightarrow \tau_{2}} d \stackrel{\Delta}{\Longleftrightarrow} x R_{\rho}^{\tau_{1}} y \supset(f @ x) R_{\rho}^{\tau_{2}}\left(d^{\circ} y\right)$, this is the standard way of defining at higher types a logical relation between typed applicative structures.

Gomard interprets types according to the informal semantics, i.e. $\llbracket b_{a s} e^{s} \rrbracket=$ const $t_{\perp}$ and $\llbracket c o d e^{s} \rrbracket=e x p_{\perp}$. According to the fundamental lemma of logical relations, if the two interpretations of each operation/constant of $P C F_{2}$ are logically related, then the two interpretations of each $P C F_{2}$-term are logically related. It is easy to do this check for all operations/constants except $\underline{\lambda}$. In the case of $\underline{\lambda}$ one can only hand wave, since the interpretation is informally given. Therefore, Gomard concludes that he has proved correctness.

Remark. Gomard does not mention explicitly logical relations. However, his definition of $R$ is given by induction on the structure of $P C F_{2}$-types, while correctness is proved by induction of the structure $P C F_{2}$-terms $\Gamma P_{P C F_{2}} e: \tau$. This is typical of logical relations.

In order to patch the proof one would have to change the definition of $R_{\rho}^{\text {code }}$, since in the intended semantics $\llbracket c o d e s \rrbracket=\exp _{\perp}^{N}$ or $(\exp \times N)_{\perp}^{N}$, and check the case of $\underline{\lambda}$ (which now has an interpretation). We doubt that this can be done, for the following reasons (for simplicity we take $\llbracket \operatorname{code}^{s} \rrbracket=e x p_{\perp}^{N}$ ): 
- The interpretation of $\underline{\lambda}$ may capture variables that ought to remain free. For instance, consider the interpretation of $x$ : code $P_{P C F_{2}} \underline{\lambda} y \cdot x:$ code, which is a function $f: e x p_{\perp}^{N} \rightarrow e x p_{\perp}^{N}$, and the element $[M]=\lambda n . u p(M)$ of $e x p_{\perp}^{N}$, then $f([M])=\lambda n . u p\left(\lambda x_{n} . M\right)$ (here there is some overloading in the use of $\lambda$, since $\lambda n$ is a semantic lambda while $\lambda x_{n}$ is syntactic). Depending on the choice of $n$ we may bind a variable free in $M$, therefore the semantics of $\underline{\lambda}$ fails to ensure freshness of $x_{n}$.

- The semantic domain $\exp _{\perp}^{N}$ has junk elements in comparison to $\exp _{\perp}$, and so there are several ways of defining $u R_{\rho}^{\text {code }} d$, e.g.

- $\forall n: N . \forall M: \exp u(n)=u p(M) \supset \llbracket M \rrbracket_{\rho}^{o}=d$

- $\exists n: N . \forall M: \exp \cdot u(n)=u p(M) \supset \llbracket M \rrbracket_{\rho}^{o}=d$

- $\exists M: \exp . \forall n: N \cdot u(n) \equiv_{\alpha} u p(M) \supset \llbracket M \rrbracket_{p}^{o}=d$

but none of them works (nor is more canonical than the others).

If there is a way to prove correctness using (Kripke) logical relations, it is likely to involve something more subtle than parameterization by $\rho: v a r \rightarrow D$.

\section{A functor category model of $\mathrm{PCF}_{2}$}

In this section we define a categorical model of $P C F_{2}$ in a Cpo-enriched functor category $\widehat{\mathcal{D}}=\mathrm{Cpo}^{\mathcal{D}^{o p}}$, where $\mathcal{D}$ is a syntactic category corresponding to $\lambda_{o}$, and the objects of $\mathcal{D}$ can be viewed as states of a name-counter. The main property of this model is that the hom-set $\widehat{\mathcal{D}}\left(\exp p^{n}\right.$,exp $)$ is isomorphic to the set of $\lambda_{0}$-terms modulo $\alpha$-conversion whose free variables are included in $\left\{x_{0}, \ldots, x_{n-1}\right\}$.

\subsection{The dynamic category}

We define $\mathcal{D}$ like the category associated to an algebraic theory (as proposed by Lawvere in [Law63]), i.e.:

- an object of $\mathcal{D}$ is a natural number; we identify a natural number $n$ with the set $\{0, \ldots, n-1\}$ of its predecessors;

- an arrow from $m$ to $n$, which we call substitution, is a function $\sigma: n \rightarrow$ $\Lambda(m)$, where $\Lambda(m)$ is the set of $\lambda_{o}$-terms modulo $\alpha$-conversion with free variables included in $\left\{x_{0}, \ldots, x_{m-1}\right\}$; thus $\mathcal{D}(m, n)=\Lambda(m)^{n}$;

- composition is given by composition of substitutions with renaming of bound variables (which is known to respect $\alpha$-conversion). Namely, for $\sigma_{1}: m \rightarrow n$ and $\sigma_{2}: n \rightarrow p$ the substitution $\left(\sigma_{2} \circ \sigma_{1}\right): m \rightarrow p$ is given by $\left(\sigma_{2} \circ \sigma_{1}\right)(i)=$ $N_{i}\left[\sigma_{1}\right]$, where $i \in p, N_{i}=\sigma_{2}(i) \in \Lambda(n), N_{i}\left[\sigma_{1}\right] \in \Lambda(m)$ is the result of applying in parallel to $N_{i}$ the substitutions $x_{j}:=M_{j}$ with $j \in m$.

Identities are given by identity substitutions id $: n \rightarrow \Lambda(n)$.

It is easy to see that $\mathcal{D}$ has finite products: the terminal object is 0 , and the product of $m$ with $n$ is $m+n$. Therefore, the object $n$ is the product of $n$ copies of the object 1 , moreover $\mathcal{D}(m, 1)=\Lambda(m)$. 
Remark. We can provide an informal justification for the choice of $\mathcal{D}$. The objects of $\mathcal{D}$ correspond to the states of a name-counter: state $m$ means that $m$ names, say $x_{0}, \ldots, x_{m-1}$, have been created so far.

For the choice of morphisms the justification is more technical: it is almost forced when one wants $\widehat{\mathcal{D}}\left(e x p^{m}, e x p\right)$ to be isomorphic to the set of $\lambda_{0}$-terms whose free variables are included in $\left\{x_{0}, \ldots, x_{m-1}\right\}$. In fact, the natural way of interpreting $\exp$ in $\widehat{\mathcal{D}}$ is with a functor s.t. $\exp (m)=$ the set of $\lambda_{0}$-terms with free names among those available at state $m$. If we require $F=Y(1)$, i.e. the image of $1 \in \mathcal{D}$ via the Yoneda embedding, and $m$ to be the product in $\mathcal{D}$ of $m$ copies of 1 , then we have $\widehat{\mathcal{D}}\left(e x p^{m}, e x p\right)=\widehat{\mathcal{D}}\left(Y(1)^{m}, Y(1)\right)=\widehat{\mathcal{D}}(Y(m), Y(1))=\mathcal{D}(m, 1)=$ $\exp (m)$. Therefore, we can conclude that $\mathcal{D}(m, n)=\exp (m)^{n}$. Moreover, to define composition in $\mathcal{D}$ we are forced to take $\lambda_{o}$-terms modulo $\alpha$-conversion.

\subsection{The static category}

We define $\widehat{\mathcal{D}}$ as the functor category $\mathrm{Cpo}^{\mathcal{D}^{o p}}$, which is a variant of the more familiar topos of presheaves Set ${ }^{\mathcal{D P}^{\mathrm{P}}}$. Categories of the form $\widehat{\mathcal{W}}$ (where $\mathcal{W}$ is a small category) have been used in [Ole85] for modeling local variables in Algollike languages. $\widehat{\mathcal{W}}$ enjoys the following properties:

- it has small limits and colimits (computed pointwise), and exponentials;

- it is Cpo-enriched, thus one can interpret fix-point combinators and solve recursive domain equations by analogy with Cpo;

- there is a full and faithful embedding $Y: \mathcal{W} \rightarrow \widehat{\mathcal{W}}$, which preserves limits and exponentials. This is basically the Yoneda embedding $Y(w)=\mathcal{W}(-, w)$.

- the functor $\Delta:$ Cpo $\rightarrow \widehat{\mathcal{W}}$ s.t. $(\Delta X)(-)=X$ has left and right adjoints.

Since $\mathcal{D}$ has a terminal object, $\Delta: \mathbf{C p o} \rightarrow \widehat{\mathcal{D}}$ is full and faithful, and its right adjoint is the global section functor $\Gamma: \widehat{\mathcal{D}} \rightarrow$ Cpo s.t. $\Gamma F=\widehat{\mathcal{D}}(1, F)=F(0)$. A description of several constructions in $\widehat{\mathcal{W}}$ relevant for denotational semantics can be found in [Ten91]. Here we recall only the definition of exponentials.

Definition 2. The exponential object $G^{F}$ in $\widehat{\mathcal{W}}$ is the functor s.t.

- $G^{F}(w)$ is the cpo of families $s \in \prod_{f: w^{\prime} \rightarrow w} \operatorname{Cpo}\left(F w^{\prime}, G w^{\prime}\right)$ ordered pointwise and satisfying the compatibility condition

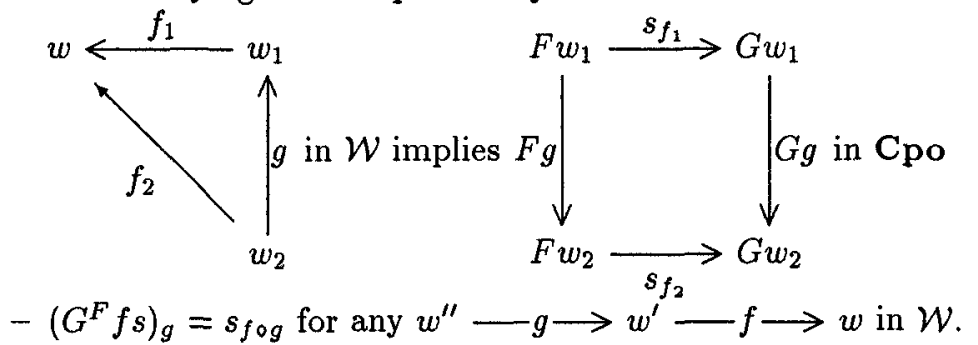

We recall also the notion of $\omega$-inductive relation in a Cpo-enriched functor category $\widehat{\mathcal{W}}$, which is used in the correctness proof. 
Definition 3. Given an object $X \in \widehat{\mathcal{W}}$, a (unary) $\omega$-inductive relation $R \subseteq X$ in $\widehat{\mathcal{W}}$ consists of a family $\left\langle R_{w} \subseteq X w \mid w \in \mathcal{W}\right\rangle$ of $\omega$-inductive relations in $\overline{\mathbf{C}}$ po satisfying the monotonicity condition:

$-f: w^{\prime} \rightarrow w$ in $\mathcal{W}$ and $x \in R_{w} \subseteq X w$ implies $X f x \in R_{w^{\prime}} \subseteq X w^{\prime}$.

\subsection{Interpretation of $P C F_{2}$}

By analogy with Section 1, we parameterize the interpretation of $P C F_{2}$ in $\widehat{\mathcal{D}}$ w.r.t. a strong monad $T$ on Cpo satisfying the additional properties stated in Note 1 . Any such $T$ induces a strong monad $T^{\mathcal{D}^{\text {op }}}$ on $\widehat{\mathcal{D}}$ satisfying the same additional properties. With some abuse of language we write $T$ for its pointwise extension $\left(T^{\mathcal{D}^{o p}} F\right)(m)=T(F(m))$.

In the proof of correctness we take $T X=X_{\perp}$, since the monad has to account only for the possibility of non-termination at specialization-time, while the interpretation of $\underline{\lambda}$ exploits only the functor category structure (and not the monad, as done for the interpretations in $\mathrm{Cpo}$ ).

Also in this case the interpretation of $P C F_{2}$ can be described by a standard translation ${ }_{-}^{s}$ into a suitable metalanguage with computational types (which play only a minor role). The key differences w.r.t. the interpretation/translation of Section 2 are: the interpretation of exp (which is not the image of a cpo via the functor $\Delta$ ), and the expression-building operation build_ $\lambda$ (which has type $(\exp \rightarrow \exp ) \rightarrow \exp$, as expected in a higher-order syntax encoding of $\left.\lambda_{o}\right)$.

- base $=T(\Delta$ (const $))$, where const is the flat cpo of ground constants. Therefore, base $(n)=T$ (const) and so global elements of base correspond to elements of the cpo $T$ (const).

- $\operatorname{code}^{s}=T(\exp )$, where $\exp =Y(1)$, i.e. the image of $1 \in \mathcal{D}$ via the Yoneda embedding $Y: \mathcal{D} \rightarrow \widehat{\mathcal{D}}$. Therefore, $\exp (n)=\Lambda(n)$ and code $(n)=T(\Lambda(n))$. It is also immediate to show that $\widehat{\mathcal{D}}\left(e x p^{n}, e x p\right)$ is isomorphic to $A(n)$ :

- $\widehat{\mathcal{D}}\left(Y(1)^{n}, Y(1)\right) \cong$ because $Y$ preserves finite products

- $\widehat{D}(Y(n), Y(1)) \cong$ because $Y$ is full and faithful

- $\mathcal{D}(n, 1) \cong \Lambda(n)$ by definition of $\mathcal{D}$.

When translating terms of $P C F_{2}$ we make use of the following expressionbuilding operations (which are interpreted by morphisms in $\widehat{\mathcal{D}}$, i.e. natural transformation):

- build_const : $\Delta$ (const) $\rightarrow$ exp s.t. build_const ${ }_{n}:$ const $\rightarrow \Lambda(n)$ is the obvious inclusion of ground constants. Alternatively, one can define build_const via the isomorphism $\widehat{\mathcal{D}}(\Delta($ const $)$, exp $) \cong \mathbf{C p o}($ const,$\Lambda(0))$ induced by the adjunction $\Delta \dashv \Gamma$.

- build_@ : exp,exp $\rightarrow \exp$ s.t.build_@ $@_{n}: \Lambda(n), \Lambda(n) \rightarrow \Lambda(n)$ is the function $M_{1}, M_{2} \mapsto M_{1} @ M_{2}$ which builds an application. Alternatively, one can define build_@ as the natural transformation corresponding to the term $x_{0} @ x_{1} \in A(2)$, via the isomorphism $\widehat{\mathcal{D}}\left(e x p^{2}, e x p\right) \cong \Lambda(2)$. There are similar definitions for build_fix and build_if.

- build_ $\lambda: e x p^{e x p} \rightarrow \exp$ is the trickiest part and is defined below. 
- the interpretation of static operations/constants is obvious, in particular we have least fixed-points because $\widehat{\mathcal{D}}$ is Cpo-enriched.

- $(\text { lift } e)^{s}=$ let $x \Leftarrow e^{s}$ in [build_const $\left.(x)\right]$

$-\underline{c}^{s}=[$ build_const $(c)]$

- $(\underline{o p} \bar{e})^{s}=$ let $\bar{M} \Leftarrow \bar{e}^{s}$ in [build_op $\left.\bar{M}\right]$, where op $\in\{f i x$, @, if $\}$

* $\underline{\underline{\lambda}}$ : code code $\rightarrow$ code is defined in terms of build_ $\lambda: \exp p^{\exp } \rightarrow \exp$ as explained below.

To define the components of the natural transformation build $\lambda: e x p^{e x p} \rightarrow \exp$ we use the following fact, which is an easy consequence of Yoneda's lemma.

Lemma 4. For any $u \in \mathcal{W}$ and $F \in \widehat{\mathcal{W}}$ there is a natural isomorphism between the functors $F^{Y(u)}$ and $F(-\times u)$.

By Lemma 4, build_ $\lambda$ amounts to a natural transformation from $\mathcal{D}(-+1,1)$ to $\mathcal{D}(-, 1)$. We describe build_ $\lambda$ through a diagram:

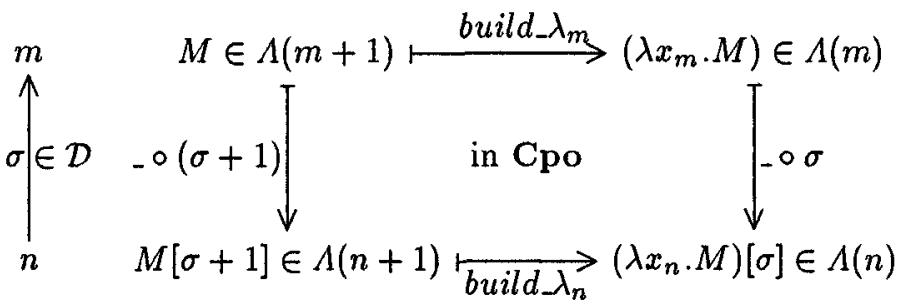

Observe that $\mathcal{D}(-, 1)=\Lambda(-)$, the substitution $(\sigma+1): m+1 \rightarrow \Lambda(n+1)$ is like $\sigma$ on $m$ and maps $m$ to $x_{n}$, while the commutativity of the diagram follows from $\left(\lambda x_{n} \cdot M[\sigma+1]\right) \equiv_{\alpha}\left(\lambda x_{m} \cdot M\right)[\sigma]$.

To define $\underline{\lambda}: T(e x p)^{T(e x p)} \rightarrow T(e x p)$ we need the following lemma.

Lemma 5. For any functor $T: \mathbf{C p o} \rightarrow \mathbf{C p o}, u \in \mathcal{W}$ and $F \in \widehat{\mathcal{W}}$ there is a natural isomorphism between the functors $(T F)^{Y(u)}$ and $T\left(F^{Y(u)}\right)$.

Proof. For any $v \in \mathcal{W}$ we give an isomorphism between $(T F)^{Y(u)}(v)$ and $T\left(F^{Y(u)}\right)(v)$ :

- $(T F)^{Y(u)}(v)=$ by Lemma 4

- $(T F)(u \times v)=$ since $T$ is extended pointwise to $\widehat{\mathcal{W}}$

- $T(F(u \times v))=$ by Lemma 4

- $T\left(F^{Y(u)}(v)\right)=$ since $T$ is extended pointwise to $\widehat{\mathcal{W}}$

$-T\left(F^{Y(u)}\right)(v)$

It is immediate to see that this family of isomorphisms is natural in $v$.

By exploiting the isomorphism $i: T(\exp )^{\exp } \rightarrow T\left(\exp p^{e x p}\right)$ given by Lemma 5 , one can define $\underline{\lambda}: T(e x p)^{T(e x p)} \rightarrow T(e x p)$ in a metalanguage with computational types as

$$
\underline{\lambda}(f)=\text { let } f^{\prime} \Leftarrow i(\lambda x: \exp . f([x])) \text { in }\left[\text { build_ } \lambda\left(f^{\prime}\right)\right]
$$


Remark. The category $\widehat{\mathcal{D}}$ has two full sub-categories $\mathcal{D}$ and $\mathbf{C p o}$, which have a natural interpretation: $\mathcal{D}$ corresponds to dynamic types, while Cpo corresponds to pure static types, i.e. those producing no residual code at specialization time (e.g. base). A key property of pure static expressions is that they cannot depend on dynamic expressions. Semantically this means that the canonical map $(\Delta X) \rightarrow(\Delta X)^{Y(u)}$, i.e. $x \mapsto \lambda y: Y(u) \cdot x$, is an isomorphism. In fact, by Lemma $4(\Delta X)^{Y(u)}$ is naturally isomorphic to $(\Delta X)(-\times u)$, which is $(\Delta X)$.

\subsection{Correctness and logical relations}

The semantics for the two-level language $P C F_{2}$ was used in [GJ91, Gom92] to prove a correctness theorem for partial evaluation. The correctness theorem relates the interpretation $I^{o}$ of the object language $\lambda_{0}$ in Cpo to the interpretation $I^{2}$ of the two-level language $P C F_{2}$ in $\hat{\mathcal{D}}$.

The first step is to define a translation ${ }_{-}^{\phi}$ from $P C F_{2}$ to $\lambda_{o}$, i.e. $\bar{x}: \bar{\tau} \vdash_{P C F_{2}}$ $e: \tau$ implies $\bar{x} \vdash_{\lambda_{0}} e^{\phi}$, which erases types and annotations, so $(\lambda x: \tau, e)^{\phi}=$ $\lambda x . e^{\phi},\left(o p_{\tau} \bar{e}\right)^{\phi}=o p \bar{e}^{\phi},(\underline{o p} \bar{e})^{\phi}=o p \bar{e}^{\phi}$ and $(\ell i f t e)^{\phi}=e^{\phi}$. By composing the translation $\phi$ with the interpretation $I^{\circ}$ we get an interpretation of $I^{1}$ of $P C F_{2}$ in Cpo, where every type is interpreted by the cpo $D=(\text { const }+(D \rightarrow D))_{\perp}$. At this stage we can state two correctness criteria (the first being a special case of the second), which exploit in an essential way the functor category structure:

- Given a closed $P C F_{2}$-expression $\emptyset \vdash e$ : code, its $I^{2}$ interpretation is a global element $d$ of $\exp _{\perp} \in \widehat{\mathcal{D}}$, and therefore $d_{0} \in \Lambda(0)_{\perp}$. Correctness for $e$ means: $d_{0}=u p(M)$ implies $\llbracket M \rrbracket^{o}=\llbracket e^{\phi} \rrbracket^{o} \in D$, for any $M \in \Lambda(0)$.

- Given an open $P C F_{2}$-expression $\bar{x}$ : code $\vdash e$ : code where $\bar{x}=x_{0}, \ldots, x_{n-1}$, its $I^{2}$ interpretation is a morphism $f: \exp _{\perp}^{n} \rightarrow \exp _{\perp}$, and therefore $f_{n}$ : $\Lambda(n)_{\perp}^{n} \rightarrow \Lambda(n)_{1}$. Correctness for $e$ means: $f_{n}\left(u p\left(x_{0}\right), \ldots, u p\left(x_{n-1}\right)\right)=u p(M)$ implies $\llbracket \bar{x} \vdash M \rrbracket^{o}=\llbracket \bar{x} \vdash e^{\phi} \rrbracket^{o}: D^{n} \rightarrow D$, for any $M \in \Lambda(n)$.

The proof of correctness requires a stronger result, which amounts to prove that the two interpretations of $P C F_{2}$ are logically related. However they live in different categories. Therefore, before one can relate them via a (Kripke) logical relation $R$ between typed applicative structures (see [MM91]), they have to be moved (via limit preserving functors) to a common category $\widehat{\mathcal{E}}$.

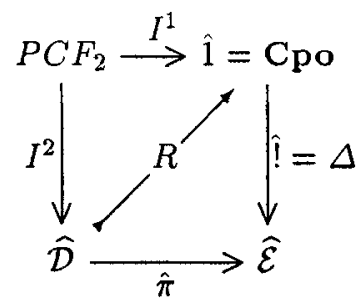

- $\mathcal{E}$ is the category whose objects are pairs $\left\langle m \in \mathcal{D}, \rho \in D^{m}\right\rangle$, while morphisms from $\langle m, \rho\rangle \rightarrow\left\langle n, \rho^{\prime}\right\rangle$ are those $\sigma: m \rightarrow n$ in $\mathcal{D}$ s.t. $\rho^{\prime}=\llbracket \sigma \rrbracket_{\rho}$

$-\pi: \mathcal{E} \rightarrow \mathcal{D}$ is the obvious projection functor $\langle m, \rho\rangle \mapsto m$. 
The Kripke logical relation $R$ is a family of $\omega$-inductive relations (see Definition 3 ) $R^{\tau}$ in $\widehat{\mathcal{E}}$ defined by induction on the structure of types $\tau$ in $P C F_{2}$.

base $R_{\langle m, p\rangle}^{\text {base }} \subset$ const $_{\perp} \times D$ s.t. $\perp R_{\langle m, \rho\rangle} d$ and $u p(c) R_{(m, \rho)} d \Leftrightarrow d=u p($ inl $c)$

code $R_{\langle m, \rho\rangle}^{\text {code }} \subset \Lambda(m)_{\perp} \times D$ s.t. $\perp R_{\langle m, \rho\rangle} d$ and $u p(M) R_{\langle m, \rho\rangle} d \stackrel{\Delta}{\Leftrightarrow} d=\llbracket M \rrbracket_{\rho}$

We must check that $R^{\text {code }}$ satisfies the monotonicity property of a Kripke relation, i.e. $\sigma:\langle m, \rho\rangle \rightarrow\left\langle n, \rho^{\prime}\right\rangle$ in $\mathcal{E}$ and $u p(M) R_{\left\langle n, \rho^{\prime}\right\rangle}^{\text {code }} d$ implies $u p(M[\sigma]) R_{\langle m, \rho\rangle}^{\text {code }} d$. This follows from $\rho^{\prime}=\llbracket \sigma \rrbracket_{\rho}$, i.e. from the definition of morphism in $\mathcal{E}$, and $\llbracket M[\sigma] \rrbracket_{\rho}=\llbracket M \rrbracket_{[\sigma]_{\rho}}$, i.e. the substitution lemma for the interpretation of $\lambda_{o}$. More diagrammatically this means

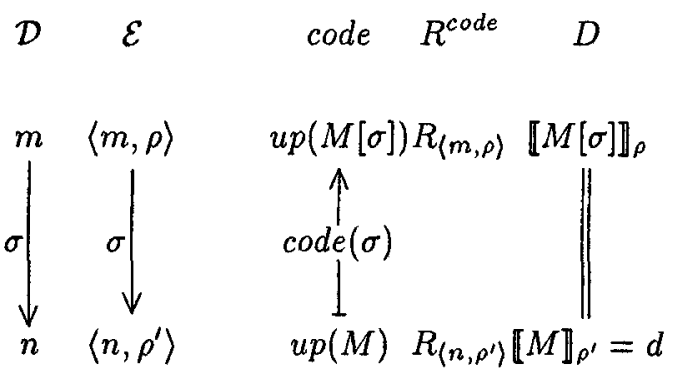

The family $R$ on functional types is defined (in the internal language) in the standard way, i.e. $f R^{\tau_{1} \rightarrow \tau_{2}} g \stackrel{\Delta}{\Leftrightarrow} \forall x, y \cdot x R^{\tau_{1}} y \supset f @^{2} x R^{\tau_{2}} g @^{1} y$, where $@^{i}$ is the binary application of the applicative structure used for the interpretation $I^{i}$. The definition of the Kripke logical relation at types base and code says that partial evaluation is only partially correct, namely if it terminates it gives the expected result.

By the fundamental lemma of logical relations, to prove that the interpretations $I^{1}$ and $I^{2}$ of $P C F_{2}$ are logically related it suffices to show that the interpretation of all higher-order constants (besides @ and $\lambda$ ) are logically related. This is a fairly straightforward check, therefore we consider only few cases, including the critical one of dynamic $\lambda$-abstraction.

@ Since $\varrho^{2}$ is strict, we need to prove only that $u p\left(M_{i}\right) R_{\langle m, \rho\rangle} d_{i}$ (for $\left.i=1,2\right)$ implies $u p\left(M_{1}\right) \varliminf^{2} u p\left(M_{2}\right) \triangleq u p\left(M_{1} @ M_{2}\right) R_{(m, \rho)} d_{1} @^{1} d_{2} \triangleq d_{1} @^{1} d_{2}$

By definition of $R$ at type code, we have to prove that $\llbracket M_{1} @ M_{2} \rrbracket_{p}=d_{1} @ 1 d_{2}$

- $\llbracket M_{i} \rrbracket_{\rho}=d_{i}$, because $u p\left(M_{i}\right) R_{\langle m, \rho\rangle} d_{i}$

- $\llbracket M_{1} @ M_{2} \rrbracket_{\rho}=@ 1\left(\llbracket M_{1} \rrbracket_{\rho}, \llbracket M_{2} \rrbracket_{\rho}\right)$, by definition of $I^{1}$

- therefore $\llbracket M_{1} @ M_{2} \rrbracket_{\rho}=d_{1} @ 1 d_{2}$

$f i x_{r}$ We need to prove that $f R^{\tau \rightarrow \tau} g$ implies $\left(\cup_{i} x_{i}\right) R^{\tau}\left(\cup_{i} y_{i}\right)$, where $x_{0}=y_{0}=\perp$ and $x_{i+1}=f @^{2} x_{i}$ and $y_{i+1}=g{ }^{1} y_{i}$.

This follows immediately from $\omega$-inductivity of $R^{r}$, i.e.

- $\perp R^{\tau} \perp$ and

- $\left(\sqcup_{i} x_{i}\right) R^{\tau}\left(\sqcup_{i} y_{i}\right)$ when $x_{i \in \omega}$ and $y_{i \in \omega}$ are $\omega$-chains and $\forall i . x_{i} R^{\tau} y_{i}$ $\omega$-inductivity of $R^{\tau}$ can be proved by a straightforward induction on $\tau$. 
$\underline{\lambda}$ The case of $\underline{\lambda}:($ code $\rightarrow$ code $) \rightarrow$ code is the most delicate one. Suppose that $f R_{\langle m, \rho\rangle}^{\text {code } \rightarrow \text { code }} g$, we have to prove that $\underline{\lambda}_{m}(f) R_{\langle m, \rho\rangle}^{\text {code }} u p\left(\operatorname{inr}\left(\lambda d: D . g @^{1} d\right)\right)$.

For this we need an explicit description of $\underline{\lambda}_{m}(f) \in \Lambda(m)_{\perp}$

- $\underline{\lambda}_{m}(f)=\perp$ when $f_{\pi: m+1 \rightarrow m}\left(u p x_{m}\right)=\perp$, where $\pi: m+1 \rightarrow m$ is the first projection in $\mathcal{D}$ and we exploit the definition of exponentials in $\widehat{\mathcal{D}}$;

- $\underline{\lambda}_{m}(f)=u p\left(\lambda x_{m} . M\right)$ when $u p(M)=f_{\pi: m+1 \rightarrow m}\left(u p x_{m}\right) \in \Lambda(m+1)_{\perp}$.

We can ignore the first case, since when $\underline{\lambda}_{m}(f)=\perp$ there is nothing to prove. In the second case, we have to prove that $\llbracket \lambda x_{m} \cdot M \rrbracket_{\rho}=u p\left(\operatorname{inr}\left(\lambda d: D . g{ }^{1} d\right)\right)$, i.e. $\llbracket M \rrbracket_{\rho[m \mapsto d]}=g \mathfrak{Q}^{1} d$ for any $d \in D$

- $u p\left(x_{m}\right) R_{\langle m+1, \rho[m \mapsto d]\rangle}^{\text {code }} d$, by definition of $R$

- $u p(M) \triangleq f_{\pi: m+1 \rightarrow m}\left(u p x_{m}\right) R_{\langle m+1, \rho[m \mapsto d]\rangle}^{\text {code }} g^{@ 1} d$, because $f R_{\langle m, \rho\rangle}^{\text {code } \rightarrow \text { code }} g$

- $\llbracket M \rrbracket_{\rho[m \mapsto d]}=g @^{1} d$, by definition of $R$.

\section{Comparisons}

In this section we make a comparative analysis of the interpretations of $P C F_{2}$ in Cpo and $\widehat{\mathcal{D}}$. In fact, to highlight more clearly the differences in the interpretations of code and dynamic $\lambda$-abstraction (and ignore orthogonal issues), it is better to work in a simplified setting, where

- $\lambda_{0}$ is the pure untyped $\lambda$-calculus;

- $P C F_{2}$ is the simply typed $\lambda$-calculus with atomic type code, and additional operations @ $:$ code, code $\rightarrow$ code and $\underline{\lambda}:($ code $\rightarrow$ code $) \rightarrow$ code .

With this simplification one can ask for total correctness of the interpretation of $P C F_{2}$ w.r.t. an interpretation of $\lambda_{o}$ in $\mathbf{C p o}$ (say in the standard model $D=$ $(D \rightarrow D)_{\perp}$ for the lazy $\lambda$-calculus). Moreover, the interpretation of $P C F_{2}$ without fix $_{\tau}$ can be given in Set or Set ${ }^{\mathcal{D p p}}$, where the syntactic category $\mathcal{D}$ has to be changed to reflect the simplifications in $\lambda_{o}$.

The following table summarizes the key differences between the original interpretation proposed by Gomard (Gomard's naive), its patching (Gomard's patched) and the interpretation in $\widehat{\mathcal{D}}$ (functor category).

\begin{tabular}{|l|c|c|c|}
\hline Semantics & Gomard's patched & Gomard's naive & functor category \\
\hline category & Set & Set & Set $^{\mathcal{D}^{\circ p}}$ \\
\hline$\llbracket$ code $\rrbracket$ & $e x p^{N}$ & $\exp$ & $\Lambda(n)$ at stage $n$ \\
\hline$\llbracket$ code $\rightarrow$ code $\rrbracket$ & $\left.\left(e x p^{N}\right)^{(e x p} p^{N}\right)$ & $e x p^{\exp }$ & $\Lambda(n+1)$ at stage $n$ \\
\hline$\llbracket \lambda \rrbracket$ & use counter & not defined & use functor category \\
\hline$R^{\text {code }}$ & not defined & $R_{\rho: N \rightarrow D}$ & $R_{n: N, p: n \rightarrow D}$ \\
\hline correctness proof & not stated & not meaningful & by Kripke log. rel. \\
\hline
\end{tabular}

Where exp is the set of $\lambda$-terms with variables in $N, \Lambda(n)$ is the set of $\lambda$-terms modulo $\alpha$-conversion with free variables in $n$, and $D \in \mathbf{C p o}$ is a domain for interpreting the lazy $\lambda$-calculus, i.e. $D=(D \rightarrow D)_{\perp}$. When describing the functor in $\widehat{\mathcal{D}}$ interpreting a certain type of $P C F_{2}$, we have given only its action on objects. The comparison shows that: 
- The functor category interpretation is very similar to Gomard's naive interpretation, when it comes to the definition of $\llbracket c o d e \rrbracket$ and $R^{\text {code }}$, though more care is taken in spelling out what object variables may occur free in an object expression.

- The advantage of working in a functor category becomes apparent in the interpretation code $\rightarrow$ code, this explains also why the functor category can handle the interpretation of $\underline{\lambda}$.

- Gomard's patched has strong similarities with the simple-minded semantics in Cpo for modeling local variables in Algol-like languages. In fact, Gomard's patched semantics parameterizes the meaning of expressions, but not that of types, w.r.t. the number of names generated used so far.

\section{Conclusions and future work}

The first part of the paper recalls the main definitions and results in [Gom92], points out the problems with the published interpretation of the two-level language $P C F_{2}$, presents possible ways of fixing the interpretation (these were proposed by Olivier Danvy, Fritz Henglein and Neil Jones during several e-mail exchanges) along the lines hinted by Gomard. After fixing the interpretation of $P C F_{2}$, there are however problems in fixing the correctness proof in [Gom92]. In the second part of the paper we propose an alternative semantics, and prove correctness for it. We have also cast doubts on the possibility of giving an interpretation of $\mathrm{PCF}_{2}$ in $\mathrm{Cpo}$ and prove its correctness w.r.t. the standard interpretation of $\lambda_{o}$ using a logical relation.

An alternative approach to correctness is proposed in [Wan93]. This avoids any explicit use of operational or denotational semantics, instead he proves correctness modulo $\beta$-conversion. Wand uses logical relations, and represents dynamic expressions using higher-order abstract syntax (while [Gom92] uses concrete syntax, and can distinguish $\alpha$-convertible expressions).

Similar problems to those pointed out in Section 2 are present in other correctness proofs (e.g. [HM94]), which adapt Gomard's approach to more complex two-level languages. We would like to test whether the functor category approach scales up to these languages.

\section{References}

[FMS96] M. Fiore, E. Moggi, and D Sangiorgi. A fully-abstract model for the picalculus. In 11th LICS Conference. IEEE, 1996.

[GJ91] K. Gomard and N. Jones. A partial evaluator for the untyped lambda calculus. J. of Func. Program., 1(1), 1991.

[Gom91] Carsten Krogh Gomard. Program Analysis Matters. PhD thesis, DIKU, November 1991. DIKU report 91/17.

[Gom92] K. Gomard. A self-applicable partial evaluator for the lambda calculus. ACM Trans. on Progr. Lang. and Systems, 14(2), 1992.

[HM94] F. Henglein and C. Mossin. Polymorphic binding-time analysis. In D. Sanella, editor, ESOP'94, volume 788 of LNCS. Springer Verlag, 1994. 
[JG593] Neil D. Jones, Carsten K. Gomard, and Peter Sestoft. Partial Evaluation and Automatic Program Generation. Prentice Hall International, 1993.

[Law63] F.W. Lawvere. Functorial semantics of algebraic theories. Proc. Nat. Acad. Sci. U.S.A., 50, 1963.

[Mit96] John C. Mitchell. Foundations of Programming Languages. The MIT Press, Cambridge, MA, 1996.

[MM91] J. Mitchell and E. Moggi. Kripke-style models for typed lambda calculus. Joumal of Pure and Applied Algebra, 51, 1991.

[Mog91] E. Moggi. Notions of computation and monads. Information and Computation, 93(1), 1991.

[Mog97a] E. Moggi. A categorical account of two-level languages. In MFPS XIII, ENTCS. Elsevier, 1997.

[Mog97b] E. Moggi. Metalanguages and applications. In Semantics and Logics of Computation, Publications of the Newton Institute. CUP, 1997.

[MW85] A. Meyer and M. Wand. Continuation semantics in typed lambda calculus. In R. Parikh, editor, Logics of Programs '85, volume 193 of LNCS. Springer Verlag, 1985.

[NN92] F. Nielson and H.R. Nielson. Two-Level Functional Languages. Number 34 in Cambridge Tracts in Theoretical Computer Science. CUP, 1992.

[Ole85] F.J. Oles. Type algebras, functor categories and block structure. In M. Nivat and J.C. Reynolds, editors, Algebraic Methods in Semantics, 1985.

[OT92] P.W. O'Hearn and R.D. Tennent. Semantics of local variables. In Applications of Categories in Computer Science, number 177 in L.M.S. Lecture Notes Series. CUP, 1992.

[PS93] A.M. Pitts and I.D.B. Stark. Observable properties of higher order functions that dynamically create local names, or: What's new? In Math. Found. of Comp. Sci. '93, volume 711 of LNCS. Springer Verlag, 1993.

[Ten91] R.D. Tennent. Semantics of Programming Languages. Prentice Hall, 1991.

[Wan93] Mitchell Wand. Specifying the correctness of binding-time analysis. Journal of Functional Programming, 3(3):365-387, July 1993. 